\title{
Cysteine String Protein Immunoreactivity in the Nervous System and Adrenal Gland of Rat
}

\author{
Sirus Azizian Kohan, ${ }^{1,2}$ Mario Pescatori, ${ }^{1}$ Nicholas C. Brecha, ${ }^{2}$ Alessandro Mastrogiacomo, ${ }^{1}$ Joy A. Umbach, \\ and Cameron B. Gundersen'
}

1Department of Molecular and Medical Pharmacology and the Crump Institute, UCLA School of Medicine, Los Angeles, California 90024 and ${ }^{2}$ Departments of Anatomy and Cell Biology, and Medicine, CURE: VA/UCLA Gastroenteric Biology Center, UCLA School of Medicine, Los Angeles, California 90024 and VAMC, West Los Angeles, California 90073

Cysteine string proteins (csps) are a recently discovered class of cysteine-rich proteins. They have been shown to associate preferentially with synaptic vesicle fractions of Torpedo electric organ or rat brain where they have been implicated in events associated with transmitter secretion. However, to date there has been no information concerning the distribution of csps in rat tissues. We investigated the localization of csps in the rat retina and CNS using immunohistochemistry with affinity purified anti-csp antibodies. Specific csp immunoreactivity having a punctate appearance is present throughout the neuraxis. Csp immunoreactivity is particularly abundant in synapse-rich regions including those of the retina, main olfactory bulb, hippocampal formation, and cerebellum. White matter tracts are devoid of csp immunoreactivity. Neuromuscular junctions show strong csp immunoreactivity. This localization of csp immunoreactivity is compatible with a role for csps in presynaptic events at a wide variety of synapses. Immunohistochemical analysis of a non-neuronal, secretory tissue, the adrenal gland, reveals prominent csp Immunoreactivity in the chromaffin cells of the adrenal medulla. However, csp immunoreactivity is not detected in adrenal cortical regions. These findings are confirmed and extended by immunoblot and Northern analyses which identify a 35 kDa and a $5 \mathrm{~kb}$ product, respectively, in extracts of adrenal. The presence of csps in the adrenal medulla suggests that these proteins may also participate in secretion-related events in certain non-neuronal cells.

[Key words: cysteine string proteins, immunohistochemistry, adrenal medulla, synaptic vesicles, presynaptic proteins, secretion, neuromuscular junction]

Cysteine string proteins (csps) were discovered as targets of a synapse-specific monoclonal antibody in Drosophila (Zinsmaier et al., 1990). Independently, the cDNA for a vertebrate csp was cloned during efforts to identify functional subunits of presynaptic calcium channels in Torpedo (Gundersen and Umbach,

Received Mar. 13, 1995; revised May 10, 1995; accepted May 16, 1995.

We thank Judy Amos for preparing the manuscript. This work was supported by grants from NIH to N.C.B. (EY04067), J.A.U. (NS31934), and C.B.G. (NS31517), by VA Medical Research funds to NCB and by Crump Institute Development funds to C.B.G., and Morphology/Imaging Core DK 41301 .

Correspondence should be adressed to Dr. C. B. Gundersen at the above address.

Copyright 1995 Society for Neuroscience 0270-6474/95/156230-09\$05.00/0
1992). Since antisense csp cRNA inhibited the expression of $\omega$-conotoxin sensitive calcium channels in Xenopus oocytes, it appeared that csps were important subunits or modulators of these channels (Gundersen and Umbach, 1992). The recent finding that csps are associated predominantly with synaptic vesicles in Torpedo (Mastrogiacomo et al., 1994b) and rat (Mastrogiacomo and Gundersen, 1995) led to the hypothesis that csps mediate a regulatory interaction between docked (or docking) synaptic vesicles and presynaptic calcium channels (Mastrogiacomo et al., 1994b).

Studies of Drosophila csp mutants have supported the conclusion that csps play an important role in the transmitter release cascade (Umbach et al., 1994; Zinsmaier et al., 1994). For instance, with a complete deletion of the Drosophila csp locus, fewer than $5 \%$ of the organisms survive to adulthood and thosc that do survive die prematurely (Zinsmaier et al., 1994). Drosophila csp mutants also display temperature-sensitive paralysis and altered electroretinograms (Zinsmaier et al., 1994). The cellular basis of these latter defects has been traced to the temperature-dependent abolition of evoked synaptic transmission (Umbach et al., 1994). This failure of transmitter release is exclusively presynaptic and arises either from an inhibition of calcium influx or from a dysfunction in the terminal steps of the exocytotic release process (Umbach et al., 1994). Thus, csps are important components of the excitation-secretion machinery at nerve terminals (Gundersen and Umbach, 1992; Gundersen et al., 1994; Mastrogiacomo et al., 1994b; Umbach et al., 1994; Zinsmaier et al., 1994).

Among different cells and organisms, there are many similarities in the proteins that participate at different stages of the vesicle targeting and secretory processes (Rothman and Orci, 1992; Bennett and Scheller 1993; Sudhof et al., 1993; FerroNovick and Jahn, 1994). For instance, several sec proteins in yeast have homologs in vertebrate secretory cells (Rothman and Orci, 1992; Bennett and Scheller, 1993; Sudhof et al., 1993). Alternatively, some proteins that were once thought to be preferentially localized to synaptic vesicles (e.g., synaptophysin; Zhong et al., 1992) are now known to be broadly distributed in other secretory pathways or to have homologs in other cell types (Sudhof et al., 1993; Ferro-Novick and Jahn, 1994). In this context, we sought to answer two questions: First, are csps widely distributed in the vertebrate nervous system and are they localized to areas that are rich in nerve terminals? If so, then they may play a central role in secretion at synapses in the vertebrate 
nervous system. Second, can csps be detected in another model secretory organ, like the adrenal gland? Our investigations indicate that csps are ubiquitously distributed in the rat nervous system and are detected at high density in many regions, including the retina, main olfactory bulb, cerehellum, and hippocampal formation. Moreover, csps are prominently expressed in chromaffin cells of the adrenal gland, but they are not detected in the steroid-secreting adrenal cortical cells. These observations imply that csps participate in excitation-secretion coupling both in neurons and in certain non-neuronal secretory cells.

\section{Materials and Methods}

Animals. Adult Sprague-Dawley rats (Harlan, San Diego, CA), weighing 180-250 gm were used in these studies. They were housed with a $12 \mathrm{hr} / 12 \mathrm{hr}$ light-dark schedule, and care and handling was in accordance with NIH guidelines.

Tissue preparation. Rats were anesthetized with $30 \%$ chloral hydrate and perfused through the heart with $0.1 \mathrm{M}$ phosphate buffer-saline $(\mathrm{pH}$ 7.4) followed by $4 \%$ paraformaldehyde (PFA) in $0.1 \mathrm{M}$ phosphate buffer ( $\mathrm{pH} 7.2$; abbreviated, $\mathrm{PB}$ ). The brain, adrenal glands, tongue, and eyes were removed. The posterior eyecup, containing the retina, and the other tissues were postfixed in 4\% PFA in PB for $2 \mathrm{hr}$ and then stored 24 $48 \mathrm{hr}$ in $25 \%$ sucrose in $\mathrm{PB}$ at $4^{\circ} \mathrm{C}$. The retina and adrenal glands were cut at $12-16 \mu \mathrm{m}$ with a cryostat, and sections were mounted onto gelatin-coated slides, dried and stored at $-20^{\circ} \mathrm{C}$ prior to immunohistochemical staining. The brain and tongue were sectioned $(35 \mu \mathrm{m})$ with a sliding microtome, and the sections were stored in $\mathrm{PB}$ at $4^{\circ} \mathrm{C}$ until immunohistochemical staining.

Anti-csp antibodies. The antibodies used for immunohistochemistry and immunoblot analysis were developed against the carboxy-terminal undecapeptide of Torpedo csp (residues 185-195) and affinity purified as described (Mastrogiacomo et al., 1994a). These antibodies show high selectivity for csps and cross-react specifically with rat csps (Mastrogiacomo et al., 1994a; Mastrogiacomo and Gundersen, 1995).

Immunohistochemical procedures. Sections of retina were processed by the indirect immunofluorescence technique. Sections were washed in $\mathrm{PB}$ and incubated overnight at $4^{\circ} \mathrm{C}$ with affinity purified antibodies against csps at $2-4 \mu \mathrm{g}-\mathrm{ml}^{-1}$ in PB containing $10 \%$ normal goat serum and $0.5 \%$ Triton X-100. Sections were washed in PB and incubated in fluorescein isothiocyanate (ГITC) conjugated goat anti-rabbit IgG (Sigma, St. Louis, MO) at a dilution of 1:50 in PB containing 0.5\% Triton $\mathrm{X}-100$ for $2 \mathrm{hr}$ at room temperature. The sections were then washed in $\mathrm{PB}$ and coverslipped in a PB glycerin mixture with $2 \% \mathrm{KI}$

Brain, tongue, and adrenal gland sections were processed by the avidin-biotin-peroxidase immunohistochemical technique. After washing with PB, sections were incubated with anti-csp antibodies as described above. Subsequently, the sections were washed with PB and incubated with biotinylated goat anti-rabbit IgG (Sigma) at a dilution of 1:50 to 1:150 for $2 \mathrm{hr}$. Another round of PB washes was followed with the biotin-peroxidase mixture (Vectastain ABC Kit, Vector Laboratories, Belmont, CA) for $2 \mathrm{hr}$. Sections were washed, incubated for $10 \mathrm{~min}$ in $3^{\prime}, 3^{\prime}$ diaminobenzidine $(\mathrm{DAB})$ in $0.1 \mathrm{M}$ Tris $(\mathrm{pH} 7.4)$ and then for $2-5$ min in DAB in $0.1 \mathrm{M}$ Tris ( $\mathrm{pH} 7.4$ ) with $0.01 \% \mathrm{H}_{2} \mathrm{O}_{2}$. All tissue sections were mounted onto gelatin-coated slides and air dried. The sections were incubated for $10 \mathrm{sec}$ in $0.05 \%$ osmium tetroxide in distilled water, dehydrated and coverslipped in ACCUMOUNT 60 Mounting Medium (Baxter Healthcare Corporation, McGaw Park, IL).

Specificity of the immunostaining was determined by incubating tissues in anti-csp antibodies that were preadsorbed with $10 \mu \mathrm{M}$ synthetic $\operatorname{csp}_{185-195}$ peptide overnight at $4^{\circ} \mathrm{C}$.

Immunoblot analysis. Whole rat adrenals were homogenized in 10 volumes of $0.25 \mathrm{M}$ sucrose and centrifuged for $10 \mathrm{~min}$ at $1000 \times \mathrm{g}$. This low-speed supernatant was centrifuged at $10,000 \times g$. The resulting pellet was suspended in sample buffer and electrophoresis and immunoblot analysis were performed as described before (Gundersen et al., 1994; Mastrogiacomo et al., 1994a,b).

Northern analysis. Poly $\left(\mathrm{A}^{+}\right)$RNA was prepared from adult rat adrenal using a chloroform-phenol extraction procedure (Gundersen et al., 1983). RNA was resolved on a $1 \%$ formaldehyde-agarose gel and transferred to nitrocellulose filter as described previously (Gundersen and Umbach, 1992). The filter was probed with a cDNA probe corresponding to the full $2.75 \mathrm{~kb}$ insert of the rat csp clone (Mastrogiacomo and Gundersen, 1995) using high stringency conditions of hybridization
(50\% formamide and $5 \times \mathrm{SSPE}$ at $42^{\circ} \mathrm{C}$ ) and washing $[0.1 \times \mathrm{SSPE}$, $0.1 \%$ SDS at $65^{\circ} \mathrm{C}$, see Gundersen and Umbach, 1992]. Bound probe was detected by autoradiography.

\section{Results}

Csp immunoreactivity is present in the neuropil of all regions of the neuraxis of adult rats (Fig. $1 A, B$; abbreviations are defined in the Appendix). White matter tracts, such as the optic tract, corpus callosum, anterior commissure, pyramidal tract, and fimbria lack csp immunoreactivity (Fig. 1A,B). No csp immunoreactivity is seen in control sections where the primary antibody was preadsorbed with the peptide antigen (Fig. 1C). High levels of csp immunoreactivity are seen in many structures (Table 1) including the retina, main and accessory olfactory bulbs, globus pallidus, substantia innominata, hippocampal formation, substantia nigra pars reticulata, superficial layers of the superior colliculus, cochlear nuclei, and cerebellum (Fig. 1A,B). In addition, csp immunoreactivity is prominent in many other areas, including the anterior olfactory nucleus, amygdala, accumbens nucleus, caudate putamen, selected thalamic nuclei, including the lateral dorsal nucleus, ventral posterolateral and posteromedial nuclei, lateral and medial geniculate nuclei, inferior colliculus, inferior olivary complex, spinal trigeminal nucleus, nucleus of the solitary tract, and the dorsal horn of spinal cord (Fig. $1 A, B)$. There is little or no csp immunoreactivity in regions highly enriched in nerve cell bodies, such as the pyramidal and granule cell layers of the hippocampal formation and the granule cell layers of the main and accessory olfactory bulb. The only exceptions are the cell bodies in the supraoptic nucleus and the paraventricular nucleus of the hypothalamus which contain high csp immunoreactivity in their cytoplasm (data not shown). Instead, the distribution of csp immunoreactivity is correlated with the density of synapses along the neuraxis. Overall, this pattern of csp immunoreactivity is similar to that reported for two other broadly distributed synaptic vesicle antigens, synapsin I and synaptophysin (DeCamilli et al., 1983; Wiedenmann and Franke, 1985; Navone et al., 1986).

\section{Regional distribution of Csp immunoreactivity}

A more-detailed investigation was made of several areas that showed a high level of csp immunoreactivity in Figure 1. Intense, specific staining for $\operatorname{csp}$ is found in the neuropil of the glomeruli and the external plexiform layer of the olfactory bulb as well as the accessory olfactory bulb (Fig. 2). Little or no csp immunoreactivity is seen in the olfactory nerve fibers overlying the glomeruli and the mitral and granule cell layers in either the main or accessory olfactory bulb (Fig. 2).

In the hippocampus (Fig. 3), strong csp immunoreactivity is seen in the CA3 and hilar regions of the dentate gyrus where immunostaining is predominantly localized to large punctate structures which arc likely to be mossy fiber boutons (Fig. 3C). Moderate csp immunoreactivity is seen throughout the strata radiatum and oriens of the CA1, CA2, CA 3 , and the stratum moleculare of the dentate gyrus (Fig. $3 A, B$ ). Csp immunoreactivity was consistently less intense in CA1 relative to CA2, CA3 and the dentate gyrus (Fig. 3). Finally, little or no csp immunoreactivity is present in the cell body layers, the stratum granulosum and stratum pyramidale (Fig. 3).

Overall, csp immunoreactivity is high in the cerebellar cortex and it is localized to regions rich in synapses (Fig. 4). The molecular layer shows strong, homogeneously distributed csp immunoreactivity. It is difficult to resolve individual immunoreac- 

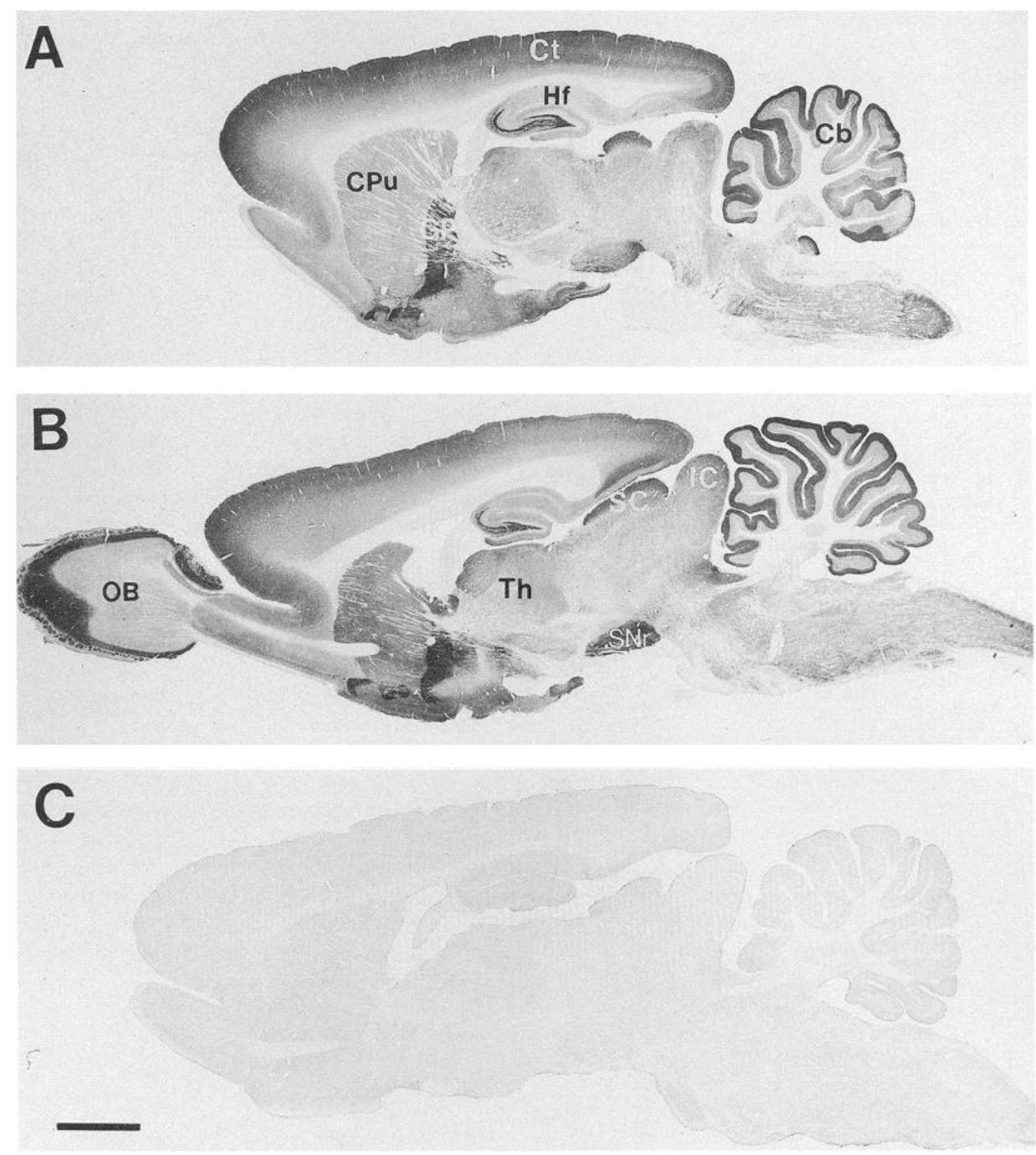

Figure 1. $A$ and $B$, Csp immunoreactivity in sagittal sections of the rat brain. High levels of immunoreactivity are present in the cerebellum $(\mathrm{Cb})$, globus pallidus $(G P)$, substantia innominata, hippocampal formation $(H f)$, main olfactory bulb $(O B)$, cochlear nuclei and substantia nigra $(S N r)$. $C$, Absence of csp immunoreactivity in a section where the anti-csp antibody was preadsorbed with the peptide antigen. Scale bar, $1500 \mu \mathrm{m}$.

tive structures in the thick frozen sections, but at high magnification the immunostaining appears to consist of small punctate structures (Fig. 4). The granule cell layer displays a more heterogeneous, intermediate level of csp immunoreactivity with large punctate structures which are mossy fiber endings in the glomeruli (Fig. 4C). No immunostaining is present in cell bodies or in the cerebellar white matter (Fig. $4 A$ ). Control sections show no csp immunoreactivity (Fig. $4 B$ ).

Csp immunoreactivity also surrounds neuronal cell bodies in many structures as seen in the cochlear nuclei (Fig. 5). In the ventral cochlear nucleus, cells are surrounded by prominent punctate csp immunoreactivity (Fig. 5) which is likely to be the large axo-somatic synapses present on cochlear neurons (Lenn and Reese, 1966; Ross and Burkel, 1971). A similar distribution of csp immunoreactivity was also seen surrounding some neuronal cell bodies in the vestibular nuclei (data not shown).

Csp immunoreactivity is detected in the retina (Fig. 6A). The synapse-rich inner and outer plexiform layers show strong immunostaining for csps (Fig. 6A). Csp immunoreactivity also surrounds some cell bodies in the ganglion cell layer and the proximal inner nuclear layer. Csp immunoreactivity is absent in the optic fiber layer, as well as the predominantly soma-rich inner and outer nuclear layers and the ganglion cell layer. No csp immunoreactivity is observed in control sections (Fig. 6B).

Csp immunoreactivity is present at neuromuscular junctions (Fig. 7). In sections of rat tongue muscle, virtually all immunostaining is confined to the terminal boutons while the muscle fibers are devoid of immunoreactivity (Fig. 7). The gradations of csp immunoreactivity at the synapse were typical of most neuromuscular junctions, and this variation may reflect the local abundance of csps at different junctional subsites.

Specific csp immunoreactivity is very strong in the adrenal medulla (Fig. 8). No immunoreactivity is detected in the adrenal cortex (Fig. 8A,B). At highe magnification (Fig. 8B,C), csp im- 


\section{Table 1. Structures containing the highest levels of CSP immunoreactivity}

Accessory olfactory bulb

\section{Cerebellum}

Cochlear nuclei (dorsal and ventral)

Globus pallidus

Hippocampus (mossy fibers)

Interpeduncular nucleus

Main olfactory bulb

Median eminence

Olivary pretectal nucleus

Retina

Spinal cord, dorsal horn

Substantia innominata

Substantia nigra, pars reticulata

Superior colliculus

munoreactivity is prominently localized to chromaffin cells of the adrenal medulla, and no immunostaining is seen in the adrenal cortex or under control conditions (Fig. $8 C$ ). These data suggest that a csp-like protein is expressed in rat adrenal medulla.

\section{Northern and immunoblot analyses of adrenal tissue}

Immunoblot analysis reveals a single immunoreactive species of about $35 \mathrm{kDa}$ in the rat adrenal $\mathrm{P}_{2}$ fraction (Fig. 9B). This 35 $\mathrm{kDa}$ antigen is absent when the primary antibody is omitted (Fig. $9 B$, lane 2). Since these same antibodies detect rat brain csp as a $35 \mathrm{kDa}$ species (Mastrogiacomo and Gundersen, 1995), we conclude that this antigen is adrenal gland csp. Northern analysis shows that a mRNA species of approximately the same size

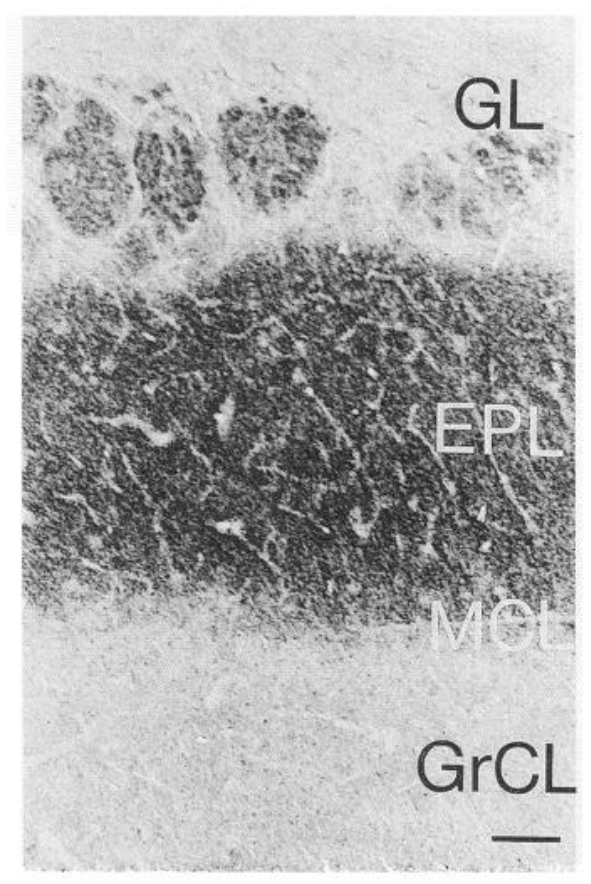

Figure 2. Csp immunoreactivity in the main olfactory bulb. The highest levels of immunoreactivity are seen in the neuropil of the glomeruli of the glomerular layer $(G L)$ and external plexiform layer $(E P L)$. Scale bar, $50 \mu \mathrm{m}$.
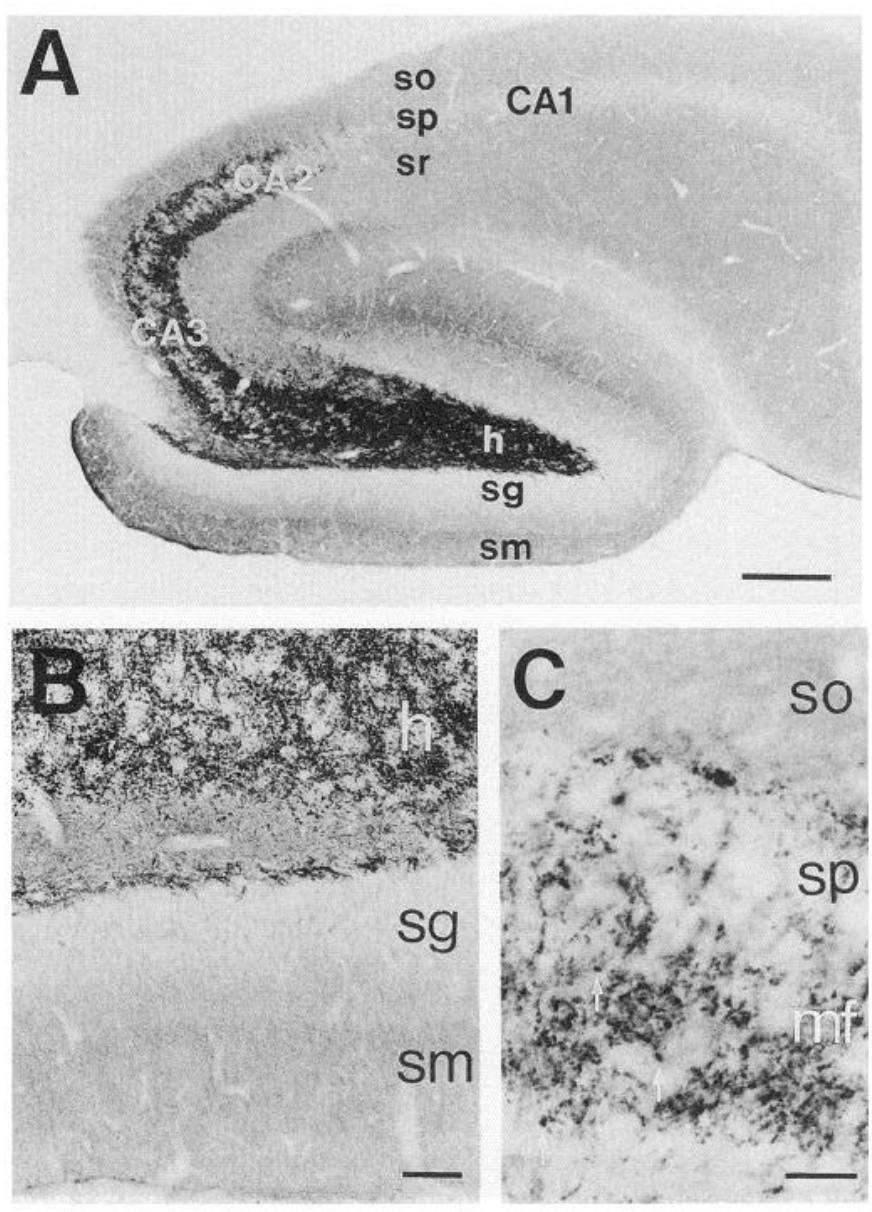

Figure 3. Csp immunoreactivity in the hippocampus. A, Csp immunoreactivity is found throughout the hippocampal formation with the highest level in mossy fibers ( $m f$; see arrows). $B$, Csp immunoreactivity in the hilus $(h)$ and stratum moleculare $(s m)$ of the dentate gyrus. $C$, The localization of csp immunoreactivity to mossy fibers ( $m f$; see $a r$ rows) in CA3 near CA2. Sagittal sections. Scale bars: $A, 250 \mu \mathrm{m} ; B$, $50 \mu \mathrm{m} ; C, 25 \mu \mathrm{m}$.

(about $5 \mathrm{~kb}$ ) as that seen in rat brain (Mastrogiacomo and Gundersen, 1995) is detected under high stringency conditions in rat adrenal (Fig. 9A). This indicates that csp mRNA is expressed in adrenal and given the results of Figure $8 B$, that csp immunoreactivity is seen in chromaffin cells, these cells are the likely source of this csp mRNA. Taken together, these immunohistochemical, immunoblot and Northern data, all indicate that csps are expressed in rat adrenal medulla.

\section{Discussion}

Csp immunoreactivity in the nervous system

Csp immunoreactivity is widely distributed in the neuropil of rat brain and retina indicating the likely presence of csps at nerve terminals. Prominent examples of csp immunoreactivity at sites of axodendritic synapses include the mossy fibers that contact pyramidal cells in the hippocampus (Laatsch and Cowan, 1966), and the mossy fibers of glomeruli in the molecular layer of the cerebellum (Gray, 1961). In contrast, csp immunoreactivity is lacking in white matter areas, and it is generally very low at sites dominated by cell bodies of neurons. The reason that csp immunoreactivity was not observed in the cell bodies, axons, or dendrites is likely to be due to the low level of csp immuno- 

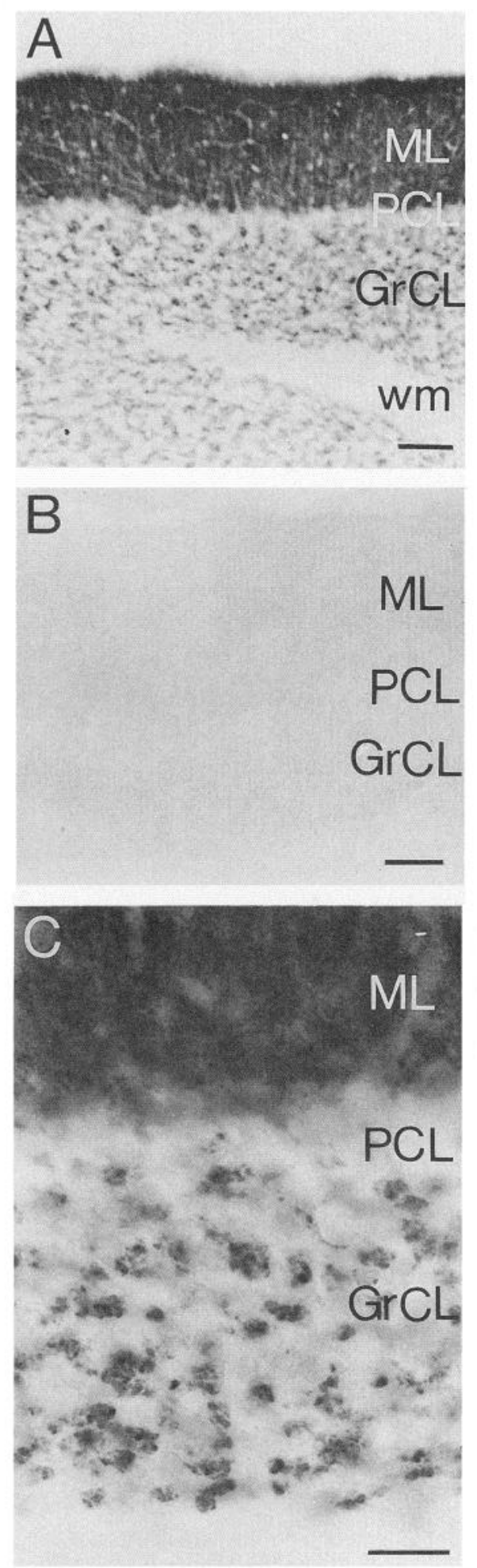

Figure 4. Csp immunoreactivity in the cerebellum. A, Immunoreactivity is highest in the molecular-cell layer $(M L)$. No immunoreactivity is present in the white matter $(w m)$. $B$, Control section illustrating the lack of immunoreactivity in a section incubated in anti-csp antibodies preadsorbed with $10^{-5} \mathrm{M} \operatorname{csp}_{184-195}$. Sagittal sections, $C$, Csp immunoreactivity is present in mossy fibers in the granule cell layer $(\mathrm{GrCL})$. Transyerse section. Scale bars: $A$ and $B, 50 \mu \mathrm{m} ; C, 25 \mu \mathrm{m}$.
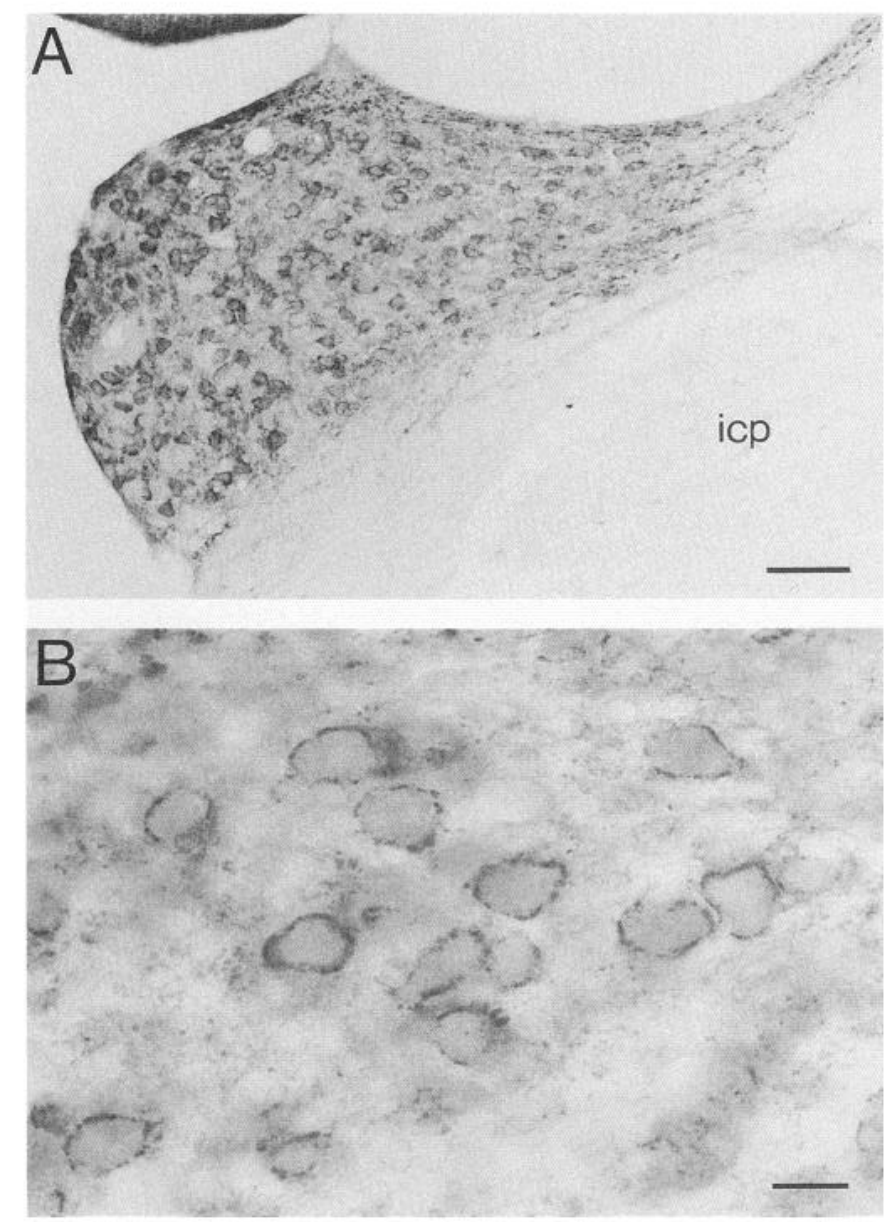

Figure 5. Csp immunoreactivity in the ventral cochlear nucleus. Csp immunoreactivity forms a halo around the soma of these neurons while the cell bodies do not stain. Punctate csp immunoreactive structures are also present in the neuropil. Scale bars: $A, 100 \mu \mathrm{m} ; B, 25 \mu \mathrm{m}$.

reactivity that is not detected by immunohistochemical methods. However, punctate, or halo-like profiles of csp immunoreactivity surround the soma of some neurons (e.g., in the cochlear nuclei) in the manner described for axosomatic synapses. Taken together, these data indicate that csps are localized to nerve terminals and are likely to be concentrated at presynaptic elements. These observations are compatible with subcellular fractionation data showing that csps are nerve ending antigens in rat brain (Mastrogiacomo and Gundersen, 1995).

In the retina, csp immunoreactivity is strongest in the inner and outer plexiform layers which have a high density of synaptic contacts. Csps were also detected at the neuromuscular junction. In nearly all respects, our results are parallel to those obtained in Drosophila where csps are highly concentrated in the retina and neuropil of the CNS and are essentially undetectable in neuronal cell bodies (Zinsmaier et al., 1990, 1994). Moreover, this pattern of csp immunoreactivity in the brain is similar to that seen for two other broadly distributed synaptic vesicle proteins, synapsin I and synaptophysin (DeCamilli et al., 1983; Wiedenmann and Franke 1985; Navone et al., 1986; Obata et al., 1986). A notable exception is the localization of csp and synaptophysin, but not synapsin immunoreactivity to the outer plexiform layer of the retina (DeCamilli et al., 1983; Wiedenmann and Franke, 1985). Thus, our data indicate that csps are relatively ubiquitous 


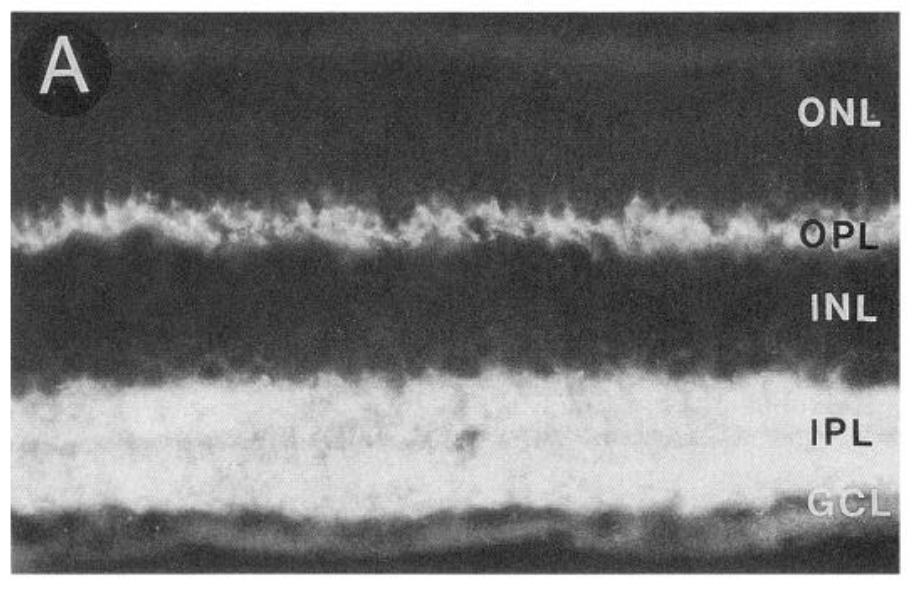

synaptic elements that are presumably associated with transmitter release sites throughout the rat nervous system.

The observed distribution of csp in the central and peripheral nervous systems is compatible with the evidence that csps are important components of the excitation-secretion machinery at a variety of nerve terminals (Gundersen et al., 1994; Mastrogiacomo et al., 1994b; Umbach et al., 1994; Zinsmaier et al., 1994). Thus, whether csps modulate presynaptic calcium channels (Gundersen and Umbach 1992; Mastrogiacomo et al., 1994b; Umbach et al., 1994), stabilize a protein complex that is necessary for release (Zinsmaier et al., 1994), or play a more direct role in catalyzing the fusion of vesicular and plasma membranes (Gundersen et al., 1994; Gundersen et al., 1995), it is essential that they be expressed presynaptically. The widespread association of csps with synaptic sites in Drosophila and rats and the physiological evidence of their importance in excitation-secretion coupling in Drosophila (Umbach et al., 1994) argues that these proteins play a crucial role in nerve terminal function.

Csp immunoreactivity was particularly strong in several regions of the rat CNS. This is exemplified by csp expression in the olfactory bulb, globus pallidus, hippocampal formation, cochlear nuclei, cerebellum, and retina. In many of these regions, the laminar organization of synapse-rich areas leads to prominent csp immunoreactivity as exemplified by the inner and outer plexiform layers in the retina and the mossy fiber boutons of the hippocampus. While other factors besides synapse density

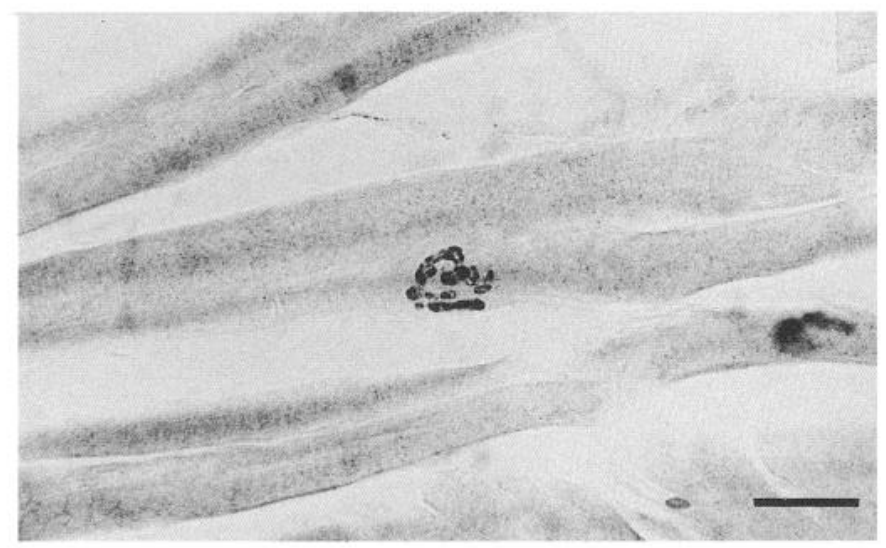

Figure 7. Csp immunoreactivity at the neuromuscular junction. This section is from the rat tongue and shows csp immunoreactivity at the terminal boutons. Neither the distal axon nor the muscle shows any detectable csp immunoreactivity. Scale bar, $50 \mu \mathrm{m}$.

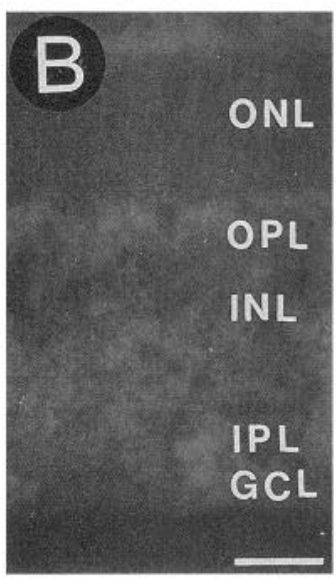

Figure 6. A, Csp immunoreactivity in the retina. Immunoreactivity is prominent in the outer plexiform layer $(O P L)$ and inner plexiform layer $(I P L) . B$, Control section illustrating the lack of csp immunoreactivity in a section incubated in anti-csp antibodies preadsorbed with $10^{-5} \mathrm{M} \mathrm{csp}_{184-195}$. Sections are cut perpendicular to the vitreal surface. Scale bar, $25 \mu \mathrm{m}$. may contribute to these staining patterns, it will be of considerable interest to ascertain whether these patterns are altered by aging, disease or manipulations that affect synaptic plasticity. For instance, in Drosophila csp mutants there is electron microscopic evidence of nerve terminal degeneration in the adult CNS (Zinsmaier et al., 1994). Ascertaining the cause of this degeneration and whether csps are implicated in vertebrate neurodegenerative disorders will be an important step for the future. Alternatively, it has recently been shown that the expression of many proteins (Fazeli et al., 1993), including synaptic vesicle proteins (Lynch et al., 1994) is modulated during hippocampal long-term potentiation. Given the abundance of csps in the mossy fiber boutons of the hippocampus, it will be interesting to determine whether there are age or activity-dependent changes in csp expression in this and other brain regions.

\section{Csp immunoreactivity in the adrenal}

While previous data (Zinsmaier et al., 1990, 1994; Mastrogiacomo et al., 1994b, Umbach et al., 1994) and results presented here support a role of csps in presynaptic function, an important question has concerned the possibility that csps might be more widely distributed in cell types other than nerve cells. For instance, among the well-characterized antigens of small synaptic vesicles, synapsin I (DeCamilli et al., 1983) is almost exclusively neuronal, while synaptophysin (Wiedenmann and Franke 1985; Navone et al., 1986; Obata et al., 1986) and p65 (Matthew et al., 1981) have also been found in endocrine and neuroendocrine structures. To address this question, we investigated whether csps were detectable in the rat adrenal medulla. Adrenal chromaffin cells produce, store and release catecholamines in response to sympathetic nerve input (von Euler, 1967). Chromaffin cells have also been widely studied as a model system for understanding the regulated exocytotic release of catecholamines (Baker and Knight, 1984; Burgoyne and Morgan, 1993). Immunocytochemical data argue compellingly that csps are expressed in these cells. Indeed, while the adrenal cortex displays only background levels of csp immunoreactivity, the adrenal medullary chromaffin cells are intensely stained. To confirm that this staining is due to the presence of csps, we conducted immunoblot and Northern analyses. Immunoblot reveals a single, specific immunoreactive protein of the appropriate mass to be csp. Northern analysis confirms that csp mRNA is present in adrenal. A single species of mRNA of about $5 \mathrm{~kb}$ is detected under high stringency conditions using a rat csp cDNA probe. The size of this mRNA is similar to that of the most prominent 


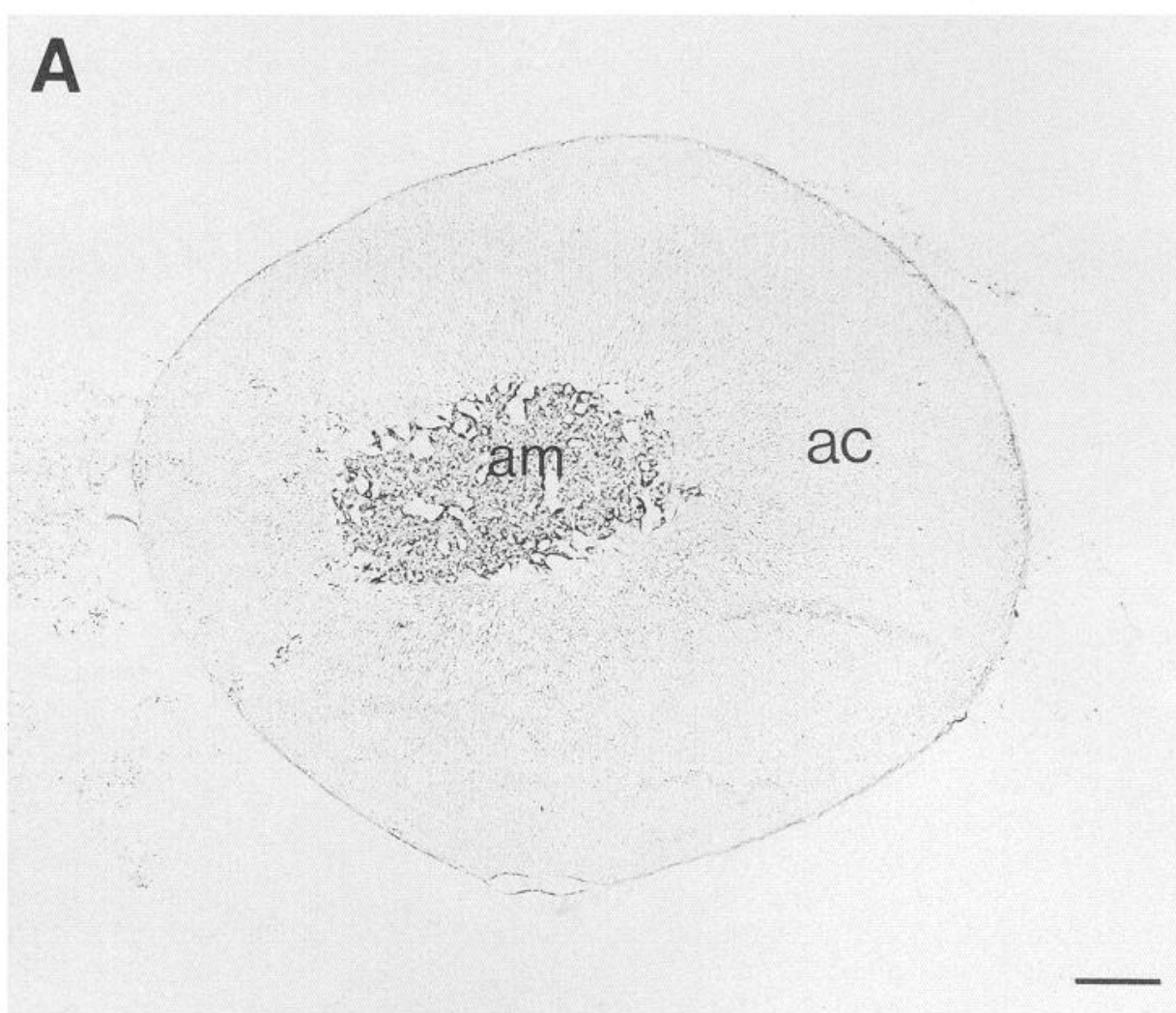

Figure 8. $A$ and $B$, Csp immunoreactivity in the adrenal gland. Intense immunoreactivity is observed in the adrenal medulla $(\mathrm{am})$, but not in cortical regions. $C$, Control section illustrating the lack of csp immunoreactivity in a section incubated in anti-csp antibodies preadsorbed with $10^{-5} \mathrm{M} \operatorname{csp}_{184-195}$ Scale bars: $A, 1500 \mu \mathrm{m} ; B$ and $C, 50$ $\mu \mathrm{m}$.

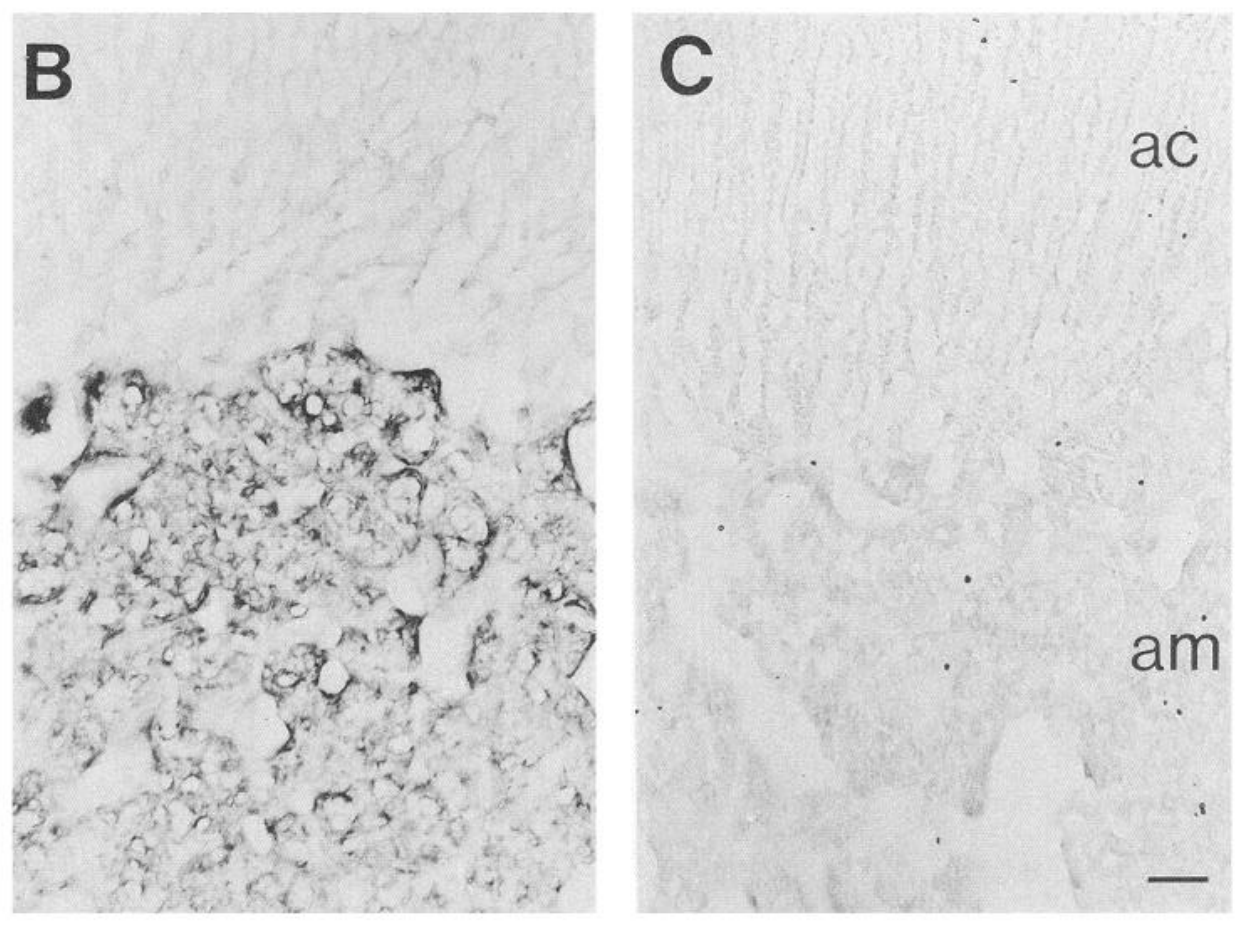

csp mRNA (5-5.5 kb) in rat brain (Mastrogiacomo and Gundersen, 1995). Taken together, these data indicate that csps are expressed in rat chromaffin cells.

What are the implications of the finding that adrenal chromaffin cells express csps? One possibility is that csps participate in a stage of the secretory cascade that is preserved both in nerve terminals and adrenal chromaffin cells. In this context it will be important to determine the subcellular distribution of csps in adrenal chromaffin cells. The reason for this derives from studies of another protein, synaptophysin. Synaptophysin is associated with small synaptic vesicles in the CNS and PNS, and it is also found in the adrenal medulla (Wiedenmann and Franke, 1985; Navone et al., 1986). However, synaptophysin does not appear to be associated with chromaffin granules in the adrenal medulla. Instead, it has been localized to small, clear vesicles of unknown function (Wiedenmann and Franke, 1985; Navone et al., 1986). 


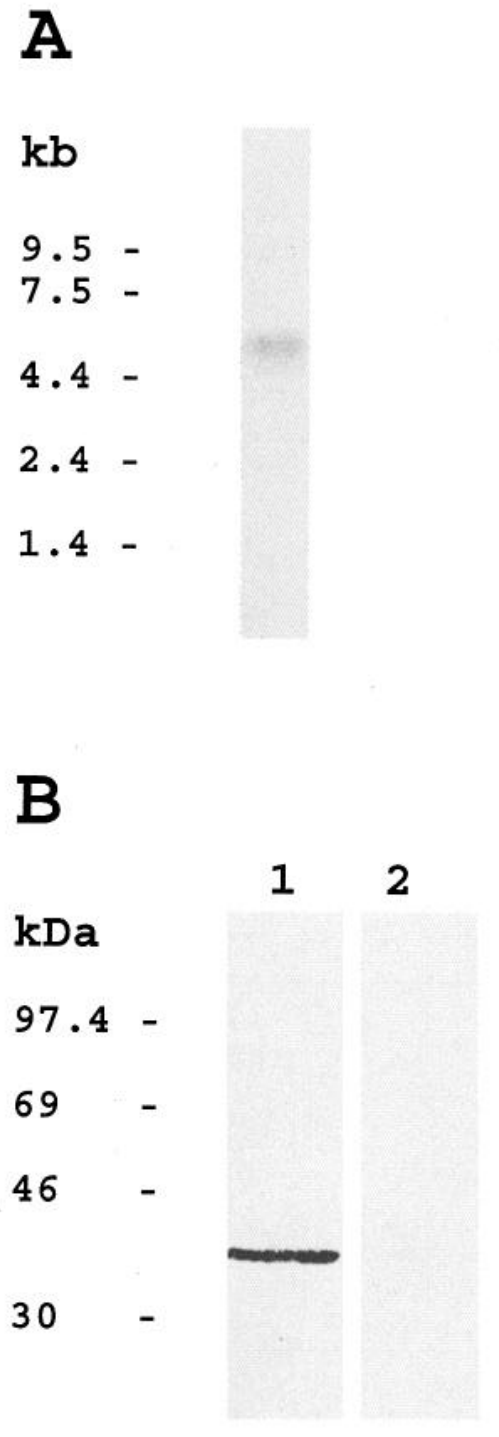

Figure 9. A, Northern analysis for csp mRNA in adrenal gland. A single RNA species is detected at about $5 \mathrm{~kb}$ under high stringency conditions. $B$, Immunoblot analysis of csp in adrenal. A single band at $35 \mathrm{kDa}$ is detected in lane 1 , but not in lane 2 where the anti-csp antibody was omitted.

More recently, these earlier findings have been the subject of debate, and there is evidence that some synaptophysin is associated with chromaffin granules (Schmidle et al., 1991). Since our immunoblot was conducted on a $\mathrm{P}_{2}$ fraction (that should be enriched in chromaffin granules), this suggests that at least some of the csp in adrenal gland is associated with chromaffin granules. However, this issue requires a more detailed investigation to resolve which membrane compartments harbor csp in adrenal. Then, given the relative accessibility of adrenal cells to studies of secretion employing either permeabilized (Knight and Baker 1982; Dunn and Holz, 1983, Wilson and Kirshner 1983) or intact cells (Neher and Marty, 1982; Schweizer et al., 1989), this system may offer useful clues to the function of csps.

\section{Appendix}

Abbreviations

ac adrenal cortex

am adrenal medulla

CA1 CA1 hippocampal subfield of Lorente de No

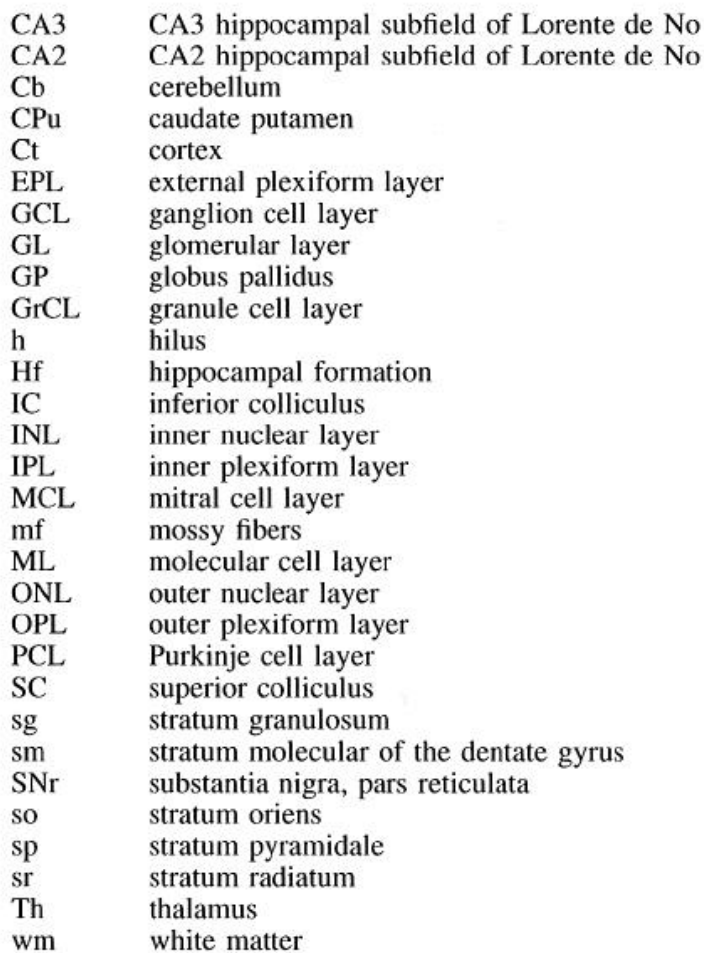

\section{References}

Baker PF, Knight DE (1984) Calcium control of exocytosis in bovine adrenal medullary chromaffin cells. Trends Neurosci 7:120-126.

Bennett MK, Scheller RH (1993) The molecular machinery for secretion is conserved from yeast to neurons. Proc Natl Acad Sci USA 90:2559-2563.

Burgoyne RD, Morgan A (1993) Regulated exocytosis. Biochem J 293: 305-316.

De Camilli P, Cameron R, Greengard P (1983) Synapsin I (Protein I), a nerve terminal specific phosphoprotein. I. Its general distribution in synapses of the central and peripheral nervous system demonstrated by immunofluorescence in frozen and plastic sections. J Cell Biol 96:1331-1354.

Dunn LA, Holz RW (1983) Catecholamine secretion from digitonintreated adrenal medullary chromaffin cells. J Biol Chem 258:9-4993.

Fazeli MS, Corbet J, Dunn MJ, Dolphin AC, Bliss TVP (1993) Changes in protein synthesis accompany long term potentiation in the dentate gyrus in vivo. J Neurosci 13:1346-1353.

Ferro-Novick S, Jahn R (1994) Vesicle fusion from yeast to man. Nature 370:191-193.

Gray EG (1961) The granule cells, mossy synapses and Purkinje spinal synapses of the cerebellum: light and electron microscopic observations. J Anat 95:345-356.

Gundersen CB, Miledi R, Parker I (1983) Glutamate and kainate receptors induced by rat brain messenger RNRA in Xenopus oocytes. Proc R Soc Lond [Biol] 220:131-140.

Gundersen CB, Umbach JA (1992) Suppression cloning of the cDNA encoding a candidate presynaptic calcium channel subunit of Torpedo. Neuron 9:527-537.

Gundersen CB, Mastrogiacomo A, Faull K, Umbach JA (1994) Extensive lipidation of a Torpedo cysteine string protein. J Biol Chem 269: 19197-19199.

Gundersen CB, Mastrogiacomo A, Umbach JA (1995) Cysteine string proteins as templates for membrane fusion: models of synaptic vesicle exocytosis. J Theor Biol 172:269-277.

Knight DE, Baker PF (1982) Calcium-dependence of catecholamine release from bovine adrenal medullary cells after exposure to intense electrical fields. J Membr Biol 68:107-140.

Laatsch RH, Cowan WM (1966) Electron microscopic studies of the dentate gyrus of the rat. J Comp Neurol 128:359-396.

Lenn NJ, Reese TS (1966) The fine structure of nerve endings in the nucleus of the trapezoid body and the ventral cochlear nucleus. Am J Anat 118:375-390.

Lynch MA, Voss KL, Rodriguez J, Bliss TVP (1994) Increase in syn- 
aptic vesicle proteins accompanies long term potentiation in the dentate gyrus. Neuroscience 60:1-5.

Mastrogiacomo A, Gundersen CB (1995) The nucleotide and deduced amino acid sequence of a rat cysteine string protein. Mol Brain Res $26: 12-18$

Mastrogiacomo A, Evans CJ, Gundersen CB (1994a) Antipeptide antibodies against a Torpedo cysteine string protein. J Neurochem 62: 873-880.

Mastrogiacomo A, Parson SM, Zampighi GA, Jenden DJ, Umbach JA, Gundersen CB (1994b) Cysteine string proteins: a potential link between synaptic vesicles and presynaptic $\mathrm{Ca}^{2+}$ channels. Science 263: 981-982.

Matthew WD, Tsavaler L, Reichardt LF (1981) Identification of a synaptic vesicle-specific membrane protein with a wide distribution in neuronal and neurosecretory tissue. J Cell Biol 91:257-269.

Navone F, Jahn R, DiGioia G, Stukenbrok K, Greengard P, DeCamilli $P$ (1986) Protein p38: an integral membrane protein specific for small vesicles of neurons and neuroendocrine cells. J Cell Biol 103 $2511-2527$

Neher E, Marty A (1982) Discrete changes of cell membrane capacitance observed under conditions of enhanced secretion in bovine adrenal chromaffin cells. Proc Natl Acad Sci USA 79:6712-6716.

Obata K Nishiye H, Fujita S, Shirao 'I, Inoue H, Uchizono K (1986) Identification of a synaptic vesicle specific 38,000 dalton protein by monoclonal antibodies. Brain Res 375:347-348.

Rothman JE, Orci L (1992) Molecular dissection of the secretory pathway. Nature 355:409-415.

Ross MD, Burkel W (1971) Flectron microscopic observations of the nucleus, glial dome and meninges of rat acoustic nerve. Am J Anat 130:73-92.
Schmidle T, Weiler R, Desnos C, Scherman D, Fischer-Colbrie R, Floor E, Winkler H (1991) Synaptin/synaptophysin, p65 and SV2: their presence in adrenal chromaffin granules and sympathetic large dense core granules. Biochim Biophys Acta 1060:251-256.

Schweizer FE, Schafer T, Tapparelli C, Grob M, Karli U, Heumann R, Thoenen H, Bookman RJ, Burger MM (1989) Inhibition of exocytosis by intracellularly applied antibodies against a chromaffin granule binding protein. Nature 339:709-712

Sudhof TC, DeCamilli P, Niemann H, Jahn R (1993) Membrane fusion machinery: insights from synaptic proteins. Cell 75:1-4.

Umbach JA, Zinsmaier KE, Eberle KK, Buchner E, Benzer S, Gundersen CB (1994) Presynaptic dysfunction in Drosophila csp mutants. Neuron 13:899-908.

von Euler US (1967) Adrenal medullary secretion and its neural control. Neuroendocrinology 2:283-384.

Wiedenmann B, Franke WW (1985) Identification and localization of synaptophysin, an integral membrane glycoprotein of $\mathrm{Mr} 38,000$ characteristic of synaptic vesicles. Cell 41:1017-1028.

Wilson SP, Kirshner N (1983) Calcium-evoked secretion from digitonin-permeabilized adrenal medullary chromaffin cells. J Biol Chem 258:4994-5000.

Zhong C, Hayzer DJ, Runge MS (1992) Molecular cloning of a cDNA encoding a novel protein related to the neuronal vesicle protein synaptophysin. Biochim Biophys Acta 1129:235-238.

Zinsmaier K, Hofbauer A, Heimbeck G, Pflugfelder GO, Buchner S, Buchner E (1990) A cysteine-string protein is expressed in retina and brain of Drosophila. J Neurogenet 7:15-29.

Zinsmaier KF, Fherle KK, Buchner F, Walter N, Benzer S (1994) Paralysis and early death in cysteine string protein mutants of Drosophila. Science 263:977-980. 